\title{
Mutual selection in time-varying networks.
}

\author{
K. Hoppe, G. J. Rodgers \\ Department of Mathematical Sciences, Brunel University, \\ Uxbridge, Middlesex UB8 3PH, United Kingdom
}

(Dated: September 19, 2013)

\begin{abstract}
Time-varying networks play an important role in the investigation of the stochastic processes that occur on complex networks. The ability to formulate the development of the network topology on the same time scale as the evolution of the random process is important for a variety of applications including the spreading of diseases. Past contributions have investigated random processes on timevarying networks with a purely random attachment mechanism. The possibility of extending these findings towards a time-varying network that is driven by mutual attractiveness is explored in this paper. Mutual attractiveness models are characterized by a linking function that describes the probability of the existence of an edge, which depends mutually on the attractiveness of the nodes on both ends of that edge. This class of attachment mechanisms has been considered before in the fitness based complex networks literature, but not on time-varying networks. Also, the impact of mutual selection is investigated alongside opinion formation and epidemic outbreaks. We find closed form solutions for the quantities of interest using a factorisable linking function. The voter model exhibits an unanticipated behavior as the network never reaches consensus in the case of mutual selection, but stays forever in its initial macroscopic configuration, which is a further piece of evidence that time-varying networks are very different from their static counterpart with respect to random processes that take place on them. We also find that epidemic outbreaks are accelerated by uncorrelated mutual selection compared to previously considered random attachment.
\end{abstract}

PACS numbers: 89.75.Hc, 87.10.Mn

\section{INTRODUCTION}

Complex networks have been studied extensively over the last decade. However, this research is usually not conducted as an end in itself, but instead aims towards a better understanding of dynamical processes that take place on a network topology. Many contributions to these epidemiological models assume that the evolution of the network and the unfoldment of the dynamical process operate on two very different time scales, such that the network evolves more slowly than the dynamical process on top of it $[1,2]$. However, this is a very restrictive assumption that does not hold in many circumstances. For a virus to spread, two individuals must be in contact when at least one of them is infected. The fact that these two individuals are linked in a static network, i.e. by friendship does not necessarily mean that the virus is passed on through their shared edge.

In recent years the field of temporal networks has attracted increasing attention and several different names have emerged in the literature, such as temporal graphs, dynamic networks, evolving graphs, time-varying graphs, ... etc. [3]. The origin of these networks comes from real world phenomena like face-to-face contacts or the phone call network, to name just a few. The central motivation is that edges are not persistent but can occur and be withdrawn within a short time. This has a direct impact on processes that take place on networks. While classical models consider the time-scale of the process to be much shorter than the time-scale of the network evolution $[2,4-6]$, the processes taking place on temporal networks have the same time-scale as fluctuations of the network topology. The issue that arises here becomes immedi- ately evident if transitivity is taken into consideration. On a static graph edges are transitive, so if for example there exists a link $(A, B)$, and a link $(A, C)$, then there exists as well an indirect path between nodes $B$ and $C$ via node $A$. In a temporal network, this transitivity no longer exists if the edges $(A, C)$ and $(A, B)$ exist during different instances of the network [3].

A temporal network and its integration can be understood in terms of the daily social life of inhabitants of a town. Each day forms an instance of a temporal network, that starts initially empty. Contacts met during the day build the links in this instance. On the following day the network instance of social contacts starts empty again. All these network instances for many days can be joined to build the integrated network, which aggregates all instances to a social graph that describes the network of acquaintances. This integrated network is what is observed if one analyzes the social graph of Facebook. However, taking the single instances into account rather than the aggregation as it is done in the classical complex networks literature adds information as outlined above. In this particular example one could think of the spread of a rumor. Modeling the spread of a rumor with the integrated, static network will bias the outcome, since the rumor will not spread across edges that are not active at the time the rumor arrives at a particular node.

An excellent overview of the various applications of temporal networks in many disciplines can be found in [3]. The applications range from person-to-person communication [7-9], cell biology and brain networks [10, 11], to aspects of distributed computing and seasonal food webs [3]. Another example of a time-varying network is the evolution of groups. In- and outflux of groups in 
conferences have been studied in $[12,13]$.

One essential difference between temporal networks and their static counterpart is the development of node centrality. Node centrality measures the importance of a node within a graph. Measuring centrality is for example used when devising an optimal vaccination strategy or designing a successful marketing campaign. In classical networks, that are driven by preferential attachment for instance, the centrality of a node is monotone, new nodes connect preferentially to central nodes in the network. It is therefore correct to assume that once a central node is found it will stay central for the life-time of the network. Therefore this node is assumed to serve well as an entry point for all sorts of campaigns. Temporal networks are very different in this respect. A node that is central in one instance can be entirely disconnected in the following time step. Therefore successful immunization strategies or information campaigns will need to take these fluctuations into account [14].

Recently, a model of a temporal fitness driven network has been investigated in [15]. Every node in that network is endowed with an intrinsic activity random variable that controls the probability that this node becomes active and initiates $m$ new links in an instance of the network. The targets of these new links are chosen at random. However, several networks have been found to be driven by mutual agreement rather than just one sided selection, the world trade network is one example [16]. An earlier example of a temporal network that is driven by mutual selection, encountering an adverse process in this topology is presented in [17]. The network model under consideration is the formation of sexual relationships and the aim of the study is to investigate the pattern of spreading diseases on this network. Pairs in this network are not chosen randomly, but with a function $\phi\left(k_{1}, k_{2}\right)$ that depends on the node degree, hence on the number of sexual relationships already existing. Diffusion and transport problems on this type of network have been studied in $[15,18]$

The present article extends the work of [15], by taking mutual preferences during the edge formation process into account. The effect of mutual selection on the topology is already well understood for static [19, 20] and dynamic networks. [21]. However, it has not yet been considered for time-varying networks. As well as the degree distribution, epidemic spread is studied here and compared to previous results in [15]. Finally, the present article extends the body of existing literature on temporal network with a study of opinion formation. The strikingly surprising result is that consensus is unobtainable in a time varying network independent of the form of the attachment kernel. The analytical results for the degree distribution and epidemic spreading are derived for the special case of a separable attachment kernel. All our analytical findings are backed by extensive numerical simulations.

\section{MODEL}

The network model consists of $N$ nodes, that are endowed with an intrinsic random fitness $x_{i}$, drawn from a probability density $\rho(x)$. Every instance of the network $\mathcal{G}_{t}$ is initialized with these $N$ nodes without edges and persists for a time span of $\Delta t$. With probability $a_{i} \Delta t$ a node $i$ becomes active and originates $m$ links, where $a_{i}$ is the activity parameter of a node, defined as $a_{i}=\eta x_{i}$, and $\eta$ is a normalization coefficient to fix the average number of active nodes per time-step to $\eta N\langle x\rangle$. With probability $1-a_{i} \Delta t$ a node remains inactive, but can receive links from other active nodes. The destination of a link depends mutually on the fitness of the origin of the link $x_{i}$ and the fitness of the destination $x_{j}$, expressed in terms of an attachment kernel $f\left(x_{i}, x_{j}\right)$.

The integrated network $G_{T}$ is the union of all network instances prior to time $T: G_{T}=\bigcup_{t=0}^{T} \mathcal{G}_{t}$. Multiple links, i.e. links that occurred during several instances are not counted several times. Merely the existence of a link in any given instance $G_{t}$ affects the existence of a link in the integrated network.

\section{A. Degree distribution}

Following the dynamics of all single instances of the network, the degree distribution of the integrated network at time $T$ is of central interest. For the final degree distribution, edges that are repeated in various instances of the network are counted as one. We will see later that the impact of these multiple edges is anyway neglectable.

The probability that a node $i$ initiates a link towards a node $j$ during $\Delta t$ is defined as

$$
\Omega_{x_{i} \rightarrow x_{j}}^{\Delta t}=a_{i} m \Delta t \cdot \frac{f\left(x_{i}, x_{j}\right)}{N \int_{0}^{\infty} f\left(x_{i}, \xi\right) \rho(\xi) d \xi} .
$$

That is the probability that node $i$ becomes active multiplied with the probability that a node with fitness $x_{i}$ initiates a link towards a node with fitness $x_{j}$. Further, denote the probability that there exists a link between nodes $i$ and $j$ in the aggregated network at time $t$ with $p_{i j}(t)$ and the probability of non-existence with $\bar{p}_{i j}(t)=1-p_{i j}(t)$. Since duplications of links during the aggregation process of all temporal instances are possible, it is straightforward to write down an equation for the probability that there exists no link between nodes $i$ and $j$, hence the following calculation is based on $\bar{p}_{i j}(t)$, which obeys this rate equation

$$
\bar{p}_{i j}(t+\Delta t)=\bar{p}_{i j}(t) \cdot\left(1-\Omega_{x_{i} \rightarrow x_{j}}^{\Delta t}\right) .
$$

Using the boundary condition $\bar{p}_{i j}(0)=1$ since all nodes are initially disconnected, one obtains

$$
\bar{p}_{i j}(t)=\exp \left\{-\frac{a_{i} m t f\left(x_{i}, x_{j}\right)}{N \int_{0}^{\infty} f\left(x_{i}, \xi\right) \rho(\xi) d \xi}\right\} .
$$


Assuming small $t / N$ which necessarily needs to fulfill $t / N \ll 1$, because the aggregated network becomes a trivial fully connected graph for $t / N$ close to 1 leads to Eq.(4). To be more precise, the average number of edges per unit of time is given by $2 m \eta\langle x\rangle$, hence the density of the integrated network at time $t$ is mostly determined by $t / N$ that must be small for a sparse network.

$$
\bar{p}_{i j}(t)=1-\frac{a_{i} m t f\left(x_{i}, x_{j}\right)}{N \int_{0}^{\infty} f\left(x_{i}, \xi\right) \rho(\xi) d \xi} .
$$

The expected out-degree of a node at time $T$ is given by

$$
k_{T}^{\text {out }}(i)=\sum_{j=0}^{N} p_{i j}(T)=T m a_{i} .
$$

Since the expected number of links that are initiated during a given time-step by a node with activity $a$ is $\Delta$ tam, $T m a_{i}$ then is the expected number of edges that originate from a node after $T / \Delta t$ time-steps. Hence multiple edges occurring during the aggregation process can be neglected.

The total degree of a node is the sum of the in-degree and the out-degree without duplications. In order to properly count, the in-degree here is similarly defined as in [15]. It is the number of edges pointing towards a node in the integrated network which have never been reciprocated. This quantity can be expressed solely in terms of $p_{i j}(t)$

$$
\begin{aligned}
k_{T}^{\text {in }}(i) & =\sum_{j=0}^{N} p_{j i}(T) \cdot \bar{p}_{i j}(T) \\
& \simeq \frac{m T}{N} \sum_{j=0}^{N} \frac{a_{j} f\left(x_{j}, x_{i}\right)}{\int_{0}^{\infty} f\left(x_{j}, \xi\right) \rho(\xi) d \xi} .
\end{aligned}
$$

The total degree of a node is then given by $k_{T}(i)=$ $k_{T}^{\text {in }}(i)+k_{T}^{\text {out }}(i)$

$$
k_{T}(i)=T m a_{i}+\frac{m T}{N} \sum_{j=0}^{N} \frac{a_{j} f\left(x_{j}, x_{i}\right)}{\int_{0}^{\infty} f\left(x_{j}, \xi\right) \rho(\xi) d \xi} .
$$

Rewriting this equation in a continuous fitness representation which is approximately correct for large $N$, one obtains

$$
k(x)=\operatorname{Tm} \eta x+\operatorname{Tm} \eta \int_{0}^{\infty} \frac{\sigma f(\sigma, x)}{\int_{0}^{\infty} f(\sigma, \xi) \rho(\xi) d \xi} \rho(\sigma) d \sigma .
$$

Assuming a factorisable form of $f(x, y)=g(x) h(y)$, Eq.(9) becomes

$$
\begin{aligned}
k(x) & =\operatorname{Tm} \eta \cdot\left(x+h(x) \cdot \frac{\langle x\rangle}{\langle h\rangle}\right) \\
\text { where }\langle h\rangle & =\int_{0}^{\infty} h(x) \rho(x) d x .
\end{aligned}
$$

The form of the degree distribution $p(k)$ depends on the choice of $f(x, y)$, respectively $g(x)$ and $h(y)$. Consider for example $f(x, y)=x y$. In this case, Eq.(10) becomes

$$
k_{T}(i)=2 T m a_{i} \Leftrightarrow x(k)=\frac{k}{2 T m \eta} .
$$

Using now that $p(k) d k \sim \rho(x) d x$

$$
\begin{aligned}
p(k) & =\rho(x(k)) \frac{d x}{d k} \\
& =\rho\left(\frac{k}{2 T m \eta}\right) \cdot \frac{1}{2 T m \eta} .
\end{aligned}
$$

Hence the form of $p(k)$ is inherited from the form of $\rho(x)$. This result is in common with the static fitness model in [20] for the case $f(x, y)=x y$. Substituting $h(x)=1$ into Eq.(10) replicates the result of [15]. In order to confirm this result, a numerical simulation was carried out, with $\rho(x)=e^{-x}, N=50,000, T=1,000, m=2$ and $\eta=0.1$. The theoretical results are in excellent agreement with the numerical results over a wide range of $k$ as Fig.( 1) shows.

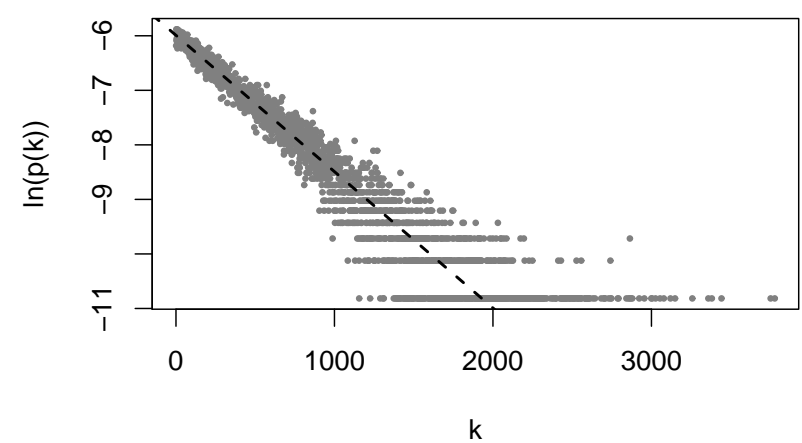

FIG. 1. Results from numerical simulation with $f(x, y)=x y$, $\rho(x)=e^{-x}, N=50,000, T=1,000, m=2$ and $\eta=0.1$, compared to the prediction (dashed-red) given in Eq.(14).

\section{B. Epidemic spreading}

This subsection is dedicated to the problem of epidemics on the network and discusses the SIS model with transition probability $\lambda$ and recovery time $1 / \mu[6]$. The quantity of interest here is the number of infected individuals at time $t$, denoted by $I^{t}$. The dynamics of this quantity can be calculated by modeling the dynamics of the number of infected individuals with activity level $a$ at time $t$, denoted with $I_{a}^{t}$.

The number of infected individuals of class $a$ obeys the 
recurrence relation

$$
\begin{aligned}
I_{a}^{t+\Delta t} & =I_{a}^{t}-\mu \Delta t I_{a}^{t} \\
& +\lambda\left(N_{a}-I_{a}^{t}\right) \cdot m a \Delta t \cdot \sum_{a^{\prime}} \frac{f\left(a, a^{\prime}\right)}{N \int_{0}^{\infty} f(a, \xi) \rho(\xi) d \xi} \cdot I_{a^{\prime}}^{t} \\
& +\lambda\left(N_{a}-I_{a}^{t}\right) \cdot \sum_{a^{\prime}} I_{a^{\prime}}^{t} m a^{\prime} \Delta t \frac{f\left(a^{\prime}, a\right)}{N \int_{0}^{\infty} f\left(a^{\prime}, \xi\right) \rho(\xi) d \xi} .
\end{aligned}
$$

With $N_{a}$ as the number of nodes in activity class $a$. The second term corresponds to the recovery of an infected individual. The third term quantifies the effect of a susceptible node to become active and connect to an infected individual. To be more precise there are $\left(N_{a}-I_{a}^{t}\right)$-many susceptible individuals with activity level $a$, each of these becomes active with probability $a \Delta t$. The term inside the sum is then the probability to connect to an individual of activity level $a^{\prime}$ that is infected. The fourth term accounts for the possibility that a susceptible node of activity level $a$ becomes infected by receiving a link from an infected node. Hence the term inside the sum corresponds to infected nodes of activity level $a^{\prime}$, that connect to node with activity $a$ and pass on the infection via this link with rate $\lambda$.

The total number of infected individuals can be obtained using

$$
I^{t}=N \int_{0}^{\infty} I_{a}^{t} \rho(a) d a
$$

The epidemic threshold is a condition on $\lambda$ and $\mu$, that represents the tipping point between an endemic and the extinction of a virus on a network. Much research has been conducted, characterizing the epidemic threshold for particular network topologies [2]. Independent of the underlying topology of the network, the epidemic threshold is given by the highest eigenvalue of the adjacency matrix of the network $[22,23]$. However, information on the adjacency matrix is not available in the present context of a mean-field approximation. A concept for the mean-field approach that follows elementary considerations of mathematical analysis has been illustrated for example in [2]. We will use this approach in the following as well.

In order to find a closed form expression for the epidemic threshold, a factorisable, not necessarily symmetric form of $f$ will be considered in the following

$$
f(x, y)=g(x) h(y)
$$

. Behind that the following definitions will be used for brevity

$$
\gamma^{t}=\sum_{a} h(a) I_{a}^{t} \quad \text { and } \quad \theta^{t}=\sum_{a} I_{a}^{t} a
$$

Using these conventions, Eq.(15) becomes

$$
\begin{aligned}
I_{a}^{t+\Delta t} & =I_{a}^{t}-\mu \Delta t I_{a}^{t} \\
& +\lambda \cdot \frac{1}{N}\left(N_{a}-I_{a}^{t}\right) m a \frac{\Delta t}{\langle h\rangle} \sum_{a^{\prime}} h\left(a^{\prime}\right) I_{a^{\prime}}^{t} \\
& +\lambda \cdot \frac{1}{N}\left(N_{a}-I_{a}^{t}\right) m \frac{h(a)}{\langle h\rangle} \Delta t \sum_{a^{\prime}} a^{\prime} I_{a^{\prime}}^{t} .
\end{aligned}
$$

The epidemic threshold can then be obtained from here without further simplifications. Summing Eq.(17) over $a$ and taking the continuous time limit, one obtains

$$
\frac{\partial I}{\partial t}=-\mu I+\lambda \gamma^{t} m \frac{\langle a\rangle}{\langle h\rangle}+\lambda \theta^{t} m .
$$

As a next step, two more equations are introduced by multiplying Eq.(17) with $a$ and summing to obtain

$$
\frac{\partial \theta}{\partial t}=-\mu \theta+\lambda \gamma^{t} m \frac{\left\langle a^{2}\right\rangle}{\langle h\rangle}+\lambda \theta \frac{\langle a h\rangle}{\langle h\rangle} m .
$$

By multiplying Eq.(17) with $h(a)$ one obtains

$$
\frac{\partial \gamma}{\partial t}=-\mu \gamma+\lambda \gamma \frac{\langle a h\rangle}{\langle h\rangle} m+\lambda \frac{\left\langle h^{2}\right\rangle}{\langle h\rangle} \theta^{t} m
$$

The resulting system of linear differential equations can be written as

$$
\left[\begin{array}{l}
\frac{\partial I}{\partial t} \\
\frac{\partial \theta}{\partial t} \\
\frac{\partial \gamma}{\partial t}
\end{array}\right]=\left[\begin{array}{ccc}
-\mu & \lambda m & \lambda \frac{\langle a\rangle}{\langle h\rangle} m \\
0 & -\mu+\lambda \frac{\langle a h\rangle}{\langle h\rangle} m & \lambda \frac{\left\langle a^{2}\right\rangle}{\langle h\rangle} m \\
0 & \lambda \frac{\left\langle h^{2}\right\rangle}{\langle h\rangle} m & -\mu+\lambda \frac{\langle a h\rangle}{\langle h\rangle} m
\end{array}\right]\left[\begin{array}{l}
I \\
\theta \\
\gamma
\end{array}\right]
$$

The solution to this matrix differential equation can be stated as a polynomial of exponentials of the eigenvalues of that matrix. Hence the value of the largest eigenvalue dominates the development of the disease, and controls whether it dies out or becomes endemic. The epidemic threshold is therefore given by

$$
\Lambda_{m}=0 .
$$

Whereby $\Lambda_{m}$ is the largest eigenvalue of above matrix. The eigenvalues are given by

$$
\begin{aligned}
& \Lambda_{1}=\lambda m \frac{\langle a h\rangle}{\langle h\rangle}-\sqrt{\frac{\left\langle h^{2}\right\rangle}{\langle h\rangle^{2}}\left\langle a^{2}\right\rangle} \cdot m \lambda-\mu \\
& \Lambda_{2}=\lambda m \frac{\langle a h\rangle}{\langle h\rangle}+\sqrt{\frac{\left\langle h^{2}\right\rangle}{\langle h\rangle^{2}}\left\langle a^{2}\right\rangle} \cdot m \lambda-\mu \\
& \Lambda_{3}=-\mu .
\end{aligned}
$$

Thus the largest eigenvalue is $\Lambda_{2}$, and hence the disease becomes endemic if

$$
\frac{\lambda}{\mu}>\frac{1}{m} \cdot \frac{\langle h\rangle}{\langle a h\rangle+\sqrt{\left\langle h^{2}\right\rangle\left\langle a^{2}\right\rangle}} .
$$




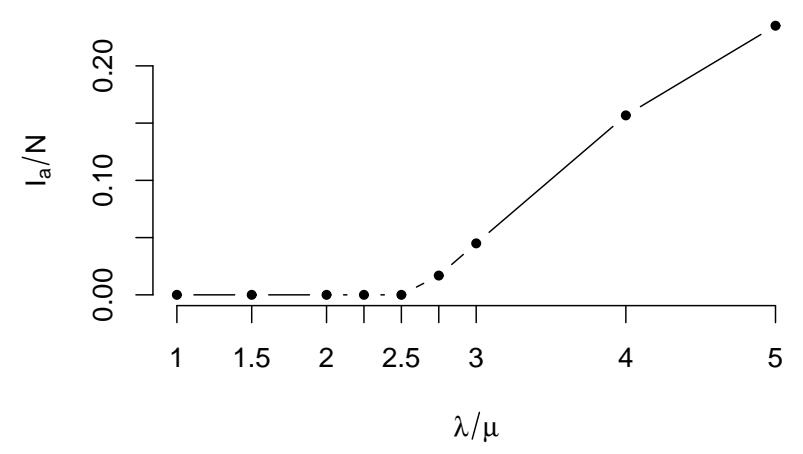

FIG. 2. Results from numerical simulation for the epidemic threshold. The plot shows the fraction of infected individuals $I / N$ vs. $\lambda / \mu$. The data is collected after $T=10^{4}$ time-steps in a network with $N=10^{4}$ nodes, averaged over 100 simulations, with $\eta=0.1, m=1, \rho(x)=e^{-x}$ and $f(x, y)=x y$. The predicted epidemic threshold from Eq.(26) is $\lambda / \mu=2.5$.

Substituting $h(a)=1$, Eq.(26) recovers the result of [15]. A numerical simulation shows as well the correctness of the above result, see Fig.(2). The reproductive number of the network can further be established in the same way as in [15], using that the expected number of edges per unit time is $m N\langle a\rangle$, leading to an average node degree of

$$
\langle k\rangle_{t}=\frac{2 E_{t}}{N}=2 m\langle a\rangle .
$$

Then the critical reproductive number $R_{0}^{c}$ is given by

$$
R_{0}^{c}=\left[\frac{\lambda}{\mu}\right]^{\mathrm{crit}} \cdot\langle k\rangle=\frac{2\langle a\rangle\langle h\rangle}{\langle a h\rangle+\sqrt{\left\langle h^{2}\right\rangle\left\langle a^{2}\right\rangle}} .
$$

To investigate the effect the mutual selection has on the epidemic properties of the network, two cases will be considered in the following: $h(a)=1$, that is the previously found result for random selection and $h(a)=a$, as the simplest form of mutuality in the selection process. The epidemic threshold for mutual selection is lower than for random selection if

$$
\frac{\langle a\rangle}{2\left\langle a^{2}\right\rangle}<\frac{1}{\langle a\rangle+\sqrt{\left\langle a^{2}\right\rangle}} .
$$

Assuming strictly positive fitness, the only solution to this inequality is given by

$$
\left\langle a^{2}\right\rangle>\langle a\rangle^{2}
$$

Hence the epidemic spreads faster in the case of mutual selection if this condition is fulfilled. Considering that the form of the degree distribution follows the form of the fitness distribution (see Eq.(14)) and knowing that real world networks exhibit very broad distributions [24], $\left\langle a^{2}\right\rangle>\langle a\rangle^{2}$ is true for many real-world networks and therefore epidemic spreading is accelerated by mutual selection.

\section{Consensus formation}

Consensus formation is another commonly considered random process, that is usually investigated on a network-type topology. Unlike results on epidemic outbreaks that were studied in the previous subsection, results for voter models on time-varying networks do not exist in the complex networks literature. However, results for the voter model on heterogeneous graphs exist $[4,5,25]$, which will serve as a benchmark. The quantity of interest in this section is the number of individuals in the network with fitness $x$ and positive opinion at a given time step. $N_{+}(x, t)$, and the traditional voter model [2] will be considered. The number of individuals with negative opinion and fitness $x$ will be denoted with $N_{-}(x, t)$.

In the traditional voter model, at every time step one node is chosen randomly and adopts the opinion of a randomly chosen neighbor. If the chosen node is not active and has not received any links during a network instance, its opinion remains the same. The number of positive opinions among nodes with fitness $x$ evolves as follows

$$
\begin{aligned}
& N_{+}(x, t+\Delta t)=N_{+}(x, t) \\
& +\rho(x) \frac{N_{-}(x, t)}{N(x)} \sum_{j=1}^{N}\left(\Omega_{x_{j} \rightarrow x}^{\Delta t}+\Omega_{x \rightarrow x_{j}}^{\Delta t}\right) \frac{N_{+}\left(x_{j}, t\right)}{N\left(x_{j}\right)} \\
& \quad-\rho(x) \frac{N_{+}(x, t)}{N(x)} \sum_{j=1}^{N}\left(\Omega_{x_{j} \rightarrow x}^{\Delta t}+\Omega_{x \rightarrow x_{j}}^{\Delta t}\right) \frac{N_{-}\left(x_{j}, t\right)}{N\left(x_{j}\right)} .
\end{aligned}
$$

The second term on the right-hand side of Eq.(31) accounts for the increase in the number of positive opinions in the class of nodes with fitness $x$. The first part of that term accounts for the probability that a randomly chosen node has fitness $x$ and negative opinion, the remainder of the term inside the sum represents the expected fraction of positive nodes in the neighborhood of $x$, which is equal to the probability of choosing a neighbor with positive opinion, since opinions are dichotomous. The third term of Eq.(31) represents the opposite process to that in the second term.

One aspect of voting consensus in temporal networks can already be inferred from Eq.(31). The consensus, if at all, is reached very slowly. The terms inside the sums of Eq.(31) are of order $1 / N^{2}$, since $\Omega_{x_{i} \rightarrow x_{j}}^{\Delta t}$ is of order $1 / N$ and $N\left(x_{j}\right)=N \rho\left(x_{j}\right)$. Hence the change of $N_{+}(x, t)$ is of order $1 / N^{3}$. Define now the probability that two nodes with fitness $x$ and $x_{j}$ are linked as

$$
\Psi\left(x, x_{j}\right)=\Omega_{x \rightarrow x_{j}}^{\Delta t}+\Omega_{x_{j} \rightarrow x}^{\Delta t}
$$

and use $N_{+}(x, t)+N_{-}(x, t)=N \rho(x)$, therefore Eq.(31) 

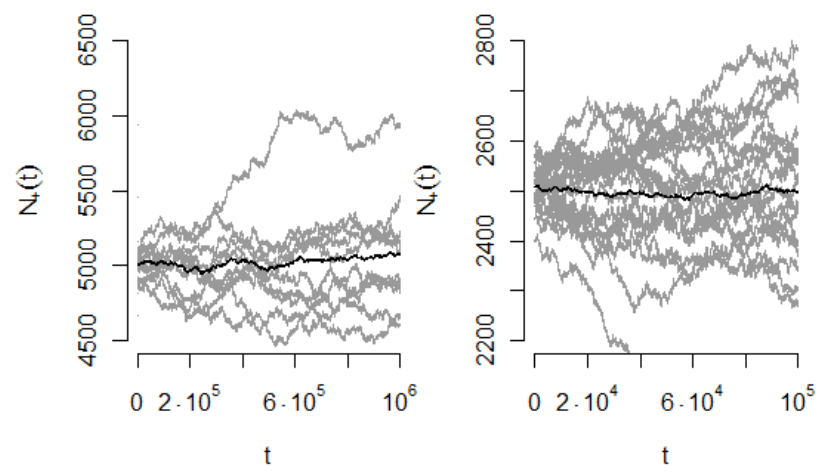

FIG. 3. Left: Traces for 10 independent simulations of $N_{+}(t)$ in grey and the average in black. The configuration of the simulation is $f(x, y)=x y, \rho(x)=e^{-x}, m=1, \eta=1 / 10$. The time horizon is $T=10^{6}$ time steps and the system contains $N=10^{4}$ nodes, with initial configuration $N_{+}(0)=5,000$. Right: Traces of 20 independent simulations of $N_{+}(t)$ in grey with average in black. The configuration is $f(x, y)=x y$, $\rho(x)=e^{-x}, m=6, \eta=1 / 10$. The time horizon is $T=$ $10^{5}$ time steps with a system size of $N=10^{4}$ and initial configuration of $N(0)=2,500$

can be rewritten to

$$
\begin{aligned}
\frac{\partial N_{+}(x, t)}{\partial t} & =\rho(x) \sum_{j=1}^{N} \Psi\left(x, x_{j}\right) \frac{N_{+}\left(x_{j}, t\right)}{N \rho\left(x_{j}\right)} \\
& -\frac{N_{+}(x, t)}{N} \sum_{j=1}^{N} \Psi\left(x, x_{j}\right) .
\end{aligned}
$$

In a steady state, $N_{+}(t)$, does not change, hence $\frac{\partial N_{+}(x, t)}{\partial t}=0$. This condition is equivalent to

$$
\rho\left(x_{i}\right) \sum_{j} \Psi\left(x_{i}, x_{j}\right) \frac{N_{+}\left(x_{j}, t\right)}{N \rho\left(x_{j}\right)}=\frac{N_{+}\left(x_{i}, t\right)}{N} \sum_{j} \Psi\left(x_{i}, x_{j}\right) .
$$

Dividing both sides by $\rho\left(x_{i}\right)$ and summing over all $i$, leads to

$$
\sum_{i} \sum_{j} \Psi\left(x_{i}, x_{j}\right) \frac{N_{+}\left(x_{j}, t\right)}{N \rho\left(x_{j}\right)}=\sum_{i} \sum_{j} \frac{N_{+}\left(x_{i}, t\right)}{N \rho\left(x_{i}\right)} \Psi\left(x_{i}, x_{j}\right) .
$$

This equation is fulfilled for any value of $t$, hence consensus can never be reached, moreover, the system stays forever in its initial configuration. Numerical simulations have been conducted to verify this result, the development of $N_{+}(t)$ for two different configurations can be found in Fig.(3). The fact that consensus can never be reached on a time-varying network shows very clearly how different this class of networks is from classical networks. Authors in [5] have shown that the time until consensus is reached $T_{N}$, starting from an equally mixed population on a complete graph is $N \ln 2$ and for heterogeneous networks with degree distribution $p(k) \propto k^{-\alpha}$ with $\alpha<3, T_{N} \ll N$. Thus consensus is usually reached reasonably fast. However, it could already been inferred from Eq.(31), that due to time-varying nature, the opinion update process is slower by several orders of magnitude compared to classic networks. That consensus is unobtainable is a surprising result.

\section{DISCUSSION}

The field of time-varying networks is still in its early developments, but is certainly of great importance for many real-world applications. The ability to formulate network evolution and a topology coupled random process on the same time-scale enhances the understanding of many real-world phenomena. The present article has introduced the concept of mutual selection on timevarying networks. Mutual selection is a concept that is already understood well for static and dynamic network models, but has not been considered on time-varying networks previously. The present study has shown that mutual selection has a direct impact on the way, epidemics spread on the network. It has also been pointed out that independent of the attachment kernel, consensus on a dichotomous decision can not be reached within the framework of a time-varying topology. One of the aims of the present article is to raise attention towards the effect of mutual selection and only the simplest random processes have been considered here. Studying more involved random processes is left for future work.
[1] A. Vespignani, Nature Physics 8, 32 (2011).

[2] A. Barrat, M. Barthlemy, and A. Vespignani, Dynamical processes on complex networks (Cambridge University Press, 2008).

[3] P. Holme and J. Saramäki, Physics reports 519, 97 (2012)

[4] V. Sood and S. Redner, Phys. Rev. Lett. 94, 178701 (2005).
[5] V. Sood, T. Antal, and S. Redner, Phys. Rev. E 77, 041121 (2008).

[6] R. Pastor-Satorras and A. Vespignani, Phys. Rev. Lett. 86, 3200 (2001).

[7] A. Vazquez, B. Rácz, A. Lukács, and A.-L. Barabási, Phys. Rev. Lett. 98, 158702 (2007).

[8] R. K. Pan and J. Saramäki, Phys. Rev. E 84, 016105 (2011). 
[9] J. L. Iribarren and E. Moro, Phys. Rev. Lett. 103, 038702 (2009).

[10] S. Lèbre, J. Becq, F. Devaux, M. Stumpf, and G. Lelandais, BMC systems biology 4, 130 (2010).

[11] J.-D. J. Han, N. Bertin, T. Hao, D. S. Goldberg, G. F. Berriz, L. V. Zhang, D. Dupuy, A. J. Walhout, M. E. Cusick, F. P. Roth, et al., Nature 430, 88 (2004).

[12] J. Stehlé, A. Barrat, and G. Bianconi, Phys. Rev. E 81, 035101 (2010).

[13] K. Zhao, J. Stehlé, G. Bianconi, and A. Barrat, Phys. Rev. E 83, 056109 (2011).

[14] S. A. Hill and D. Braha, Phys. Rev. E 82, 046105 (2010).

[15] N. Perra, B. Gonçalves, R. Pastor-Satorras, and A. Vespignani, Scientific reports 2 (2012).

[16] D. Garlaschelli and M. I. Loffredo, Phys. Rev. Lett. 93, 188701 (2004).

[17] M. Kretschmar and M. Morris, Mathematical biosciences 133, 165 (1996).

[18] S.-Y. Liu, A. Baronchelli, and N. Perra, Phys. Rev. E 87, 032805 (2013).
[19] G. Caldarelli, A. Capocci, P. De Los Rios, and M. Muñoz, Physical review letters 89, 258702 (2002).

[20] V. D. Servedio, G. Caldarelli, and P. Butta, Physical Review E 70, 056126 (2004).

[21] I. E. Smolyarenko, K. Hoppe, and G. J. Rodgers, Phys. Rev. E 88, 012805 (2013).

[22] Y. Wang, D. Chakrabarti, C. Wang, and C. Faloutsos, in Reliable Distributed Systems, 2003. Proceedings. 22nd International Symposium on (2003) pp. 25-34.

[23] D. Chakrabarti, Y. Wang, C. Wang, J. Leskovec, and C. Faloutsos, ACM Transactions on Information and System Security (TISSEC) 10, 1 (2008).

[24] R. Albert and A.-L. Barabási, Rev. Mod. Phys. 74, 47 (2002).

[25] P. Moretti, S. Liu, A. Baronchelli, and R. PastorSatorras, The European Physical Journal B 85, 1 (2012). 